\title{
GDQ METHOD FOR NATURAL CONVECTION IN A CUBIC CAVITY USING VELOCITY-VORTICITY FORMULATION
}

\author{
D. C. Lo, D. L. Young, and K. Murugesan \\ Department of Civil Engineering and Hydrotech Research Institute, \\ National Taiwan University, Taipei, Taiwan
}

Natural convection in a differentially heated cubic enclosure is studied by solving the velocity-vorticity form of the Navier-Stokes equations by a generalized differential quadrature (GDQ) method. The governing equations in the form of velocity Poisson equations, vorticity transport equations, and energy equation are solved using a coupled numerical scheme via a single global matrix for velocities, vorticities, and temperature. Vorticity and velocity coupling at the solid boundaries is enforced through a higher-order approximation by the GDQ method, thus assuring accurate satisfaction of the continuity equation. Nusselt numbers computed for $R a=10^{3}, 10^{4}, 10^{5}$, and $10^{6}$ show good agreement with the benchmark results. A mesh independence study indicates that the present numerical procedure requires much coarse mesh compared to other numerical schemes to produce the benchmark solutions of the flow and heat transfer problems.

\section{INTRODUCTION}

Vortex-dominant flow is an important characteristic of natural convection in differentially heated cavities. In situations where the main concern is heat transfer rather than the pressure field, the velocity-vorticity form of the momentum equations is a better choice compared to the primitive-variable form to achieve the divergence-free velocity field constraint for the incompressible Navier-Stokes equations in three dimensions. In addition, the vortex flow-dominated natural-convection flow field can be simulated directly by the velocity-vorticity formulation, without the need to handle the pressure term. For the case of incompressible fluid flows, if the incompressibility condition imposed by the continuity equation is satisfied by some means, then a divergence-free flow field can be computed by solving the velocityvorticity equations [1-5]. Mallinson and de Vahl Davis [6] were the first to propose the vorticity-stream function formulation without the pressure term, to study natural convection in a rectangular box. Though this formulation is easy to implement for the case of two-dimensional problems, an extension to three-dimensional problems is not straightforward. Further, the velocity, which is required for computing

Received 24 August 2004; accepted 5 February 2005.

We acknowledge financial support from the National Science Council of Taiwan through Grant NSC-93-E-002-017, and it is gratefully appreciated.

Address correspondence to D. L. Young, Department of Civil Engineering and Hydrotech Research Institute, National Taiwan University, Taipei, Taiwan. E-mail: dlyoung@ntu.edu.tw 


\begin{tabular}{|c|c|c|c|}
\hline \multicolumn{4}{|c|}{ NOMENCLATURE } \\
\hline$g$ & acceleration due to gravity & $x, y, z$ & dimensionless Cartesian coordinate \\
\hline$L$ & length & & directions \\
\hline \multirow[t]{2}{*}{$\mathrm{Nu}_{\text {mean }}$} & mean Nusselt number throughout the & $\alpha$ & diffusion coefficient \\
\hline & cavity & $\beta$ & thermal expansion coefficient \\
\hline \multirow[t]{2}{*}{$\mathrm{Nu}_{\text {overall }}$} & overall Nusselt number on the & $\Gamma$ & boundary of the closed domain \\
\hline & boundary at $x=-0.5$ or $x=0.5$ & $\mu$ & dynamic viscosity \\
\hline $\operatorname{Pr}$ & Prandtl number & $v$ & kinematic viscosity \\
\hline $\mathrm{Ra}$ & Rayleigh number & $\rho$ & mass density \\
\hline $\mathrm{Re}$ & Reynolds number & $\xi, \eta, \zeta$ & dimensionless vorticity in the $x, y, z$ \\
\hline$T$ & temperature & & directions \\
\hline$T_{\alpha}$ & reference temperature & $\Omega$ & computational domain \\
\hline$u, v, w$ & $\begin{array}{l}\text { dimensionless velocity in the } x, y, z \\
\text { directions }\end{array}$ & & \\
\hline
\end{tabular}

the Nusselt number, has to be computed as a derived variable. Wong and Baker [5] used the velocity-vorticity equations to study natural convection in a cubic enclosure. However, the two main issues encountered with the velocity-vorticity formulation are (1) that the number of variables is increased from four to six as compared to the primitive-variable form for three-dimensional problems, and (2) enforcing the vorticity definition at the solid boundaries to satisfy the continuity equation [7]. As a solution to the first problem, Davis and Carpenter [4] solved only three governing equations by considering two velocities and one vorticity as the primitive variables and computed the remaining three field variables as secondary variables. However, they handled the convective part of the governing equations using a predictor-corrector scheme, thus deviating not much from the existing algorithms for treating the pressure term.

With regard to the second problem, enforcing the vorticity definition at the wall, a higher-order scheme is essential to compute the boundary vorticity values in terms of the velocity gradients. Wong and Baker [5] used a second-orderaccurate Taylor's series expansion to compute the vorticity values at the boundaries. Davis and Carpenter [4] used an integral approach for vorticity definition at the boundary, as followed by Guevremont et al. [10]. Such low-order schemes have been commonly employed with the finite-element methods $[4,5,8,9]$ and the finite-difference methods [2,3] in the solution of velocity-vorticity equations. Since the finite-difference method and the finite-element method use only low-order approximations for spatial discretization of the differential operators, a large number of grid points have to be used to achieve benchmark solutions. Though higher-order elements can be used in the case of the finite-element method, the implementation becomes unwieldy for the case of three-dimensional flow problems. One way to overcome both the problems discussed above is to use a higher-order approximating scheme such as the generalized differential quadrature (GDQ) method.

The GDQ method approximates a partial differential equation with a higherorder polynomial by using all the grid points in the coordinate direction [11]. Hence, even with a coarse mesh, the vorticity boundary values can be evaluated with 
higher-order accuracy naturally, without the need for a separate approximation scheme. Though the GDQ method has been used to solve the Navier-Stokes equations in two and three dimensions, works are available only for the stream functionvorticity and primitive-variable forms of the Navier-Stokes equations [12-14]. Further, most of the works on 3-D natural convection, except Mallinson and de Vahl Davis [6] and Wong and Baker [5], used the primitive-variable form of the Navier-Stokes equations. Numerical solutions for natural convection in a cubic cavity have also been reported using numerical schemes based on a pseudo-spectral Chebyshev algorithm [15], the lattice Boltzmann model [16], the finite-difference method [17], the finite-element method [5], etc. Wakashima and Saitoh [18] used a vorticity-vector potential formulation to produce benchmark solutions for natural convection in a cubic cavity using uniform grids of size $120^{3}$. All the above numerical procedures employed finer meshes of the order of $51^{3}$ and above in order to achieve benchmark solutions for $10^{3} \leq \mathrm{Ra} \leq 10^{6}$. Wong and Baker [5] used a mesh of size $48^{3}$ to solve the velocity-vorticity equations by the finite-element method using a parallel computational algorithm. Recently, Lo et al. [19] reported results for natural convection in a square cavity using a simple numerical algorithm based on the velocity-vorticity formulation and the GDQ method for $\mathrm{Ra}=10^{3}-10^{6}$. They demonstrated that the use of the GDQ method in combination with the velocity-vorticity formulation requires only a much coarser mesh compared to other numerical schemes to achieve the benchmark solutions. In the present work, a numerical scheme based on the GDQ method is proposed to solve the velocity-vorticity form of the Navier-Stokes equations with the aim of offseting the increased computational effort by the increased number of field variables for three-dimensional problems in the velocity-vorticity formulation. The methodology underlying the 2-D model of the velocity-vorticity formulation using the GDQ method developed by Lo et al. [19] is now extended to 3-D work.

All the flow variables and the temperature scalar are coupled together through their coefficient matrices resulting from the approximation of the governing equations by the GDQ method. The time derivatives in the governing equations are discretized using a second-order-accurate, implicit, three-time-level scheme. The final simultaneous equations are solved using a BiCG iterative scheme by storing only the nonzero entries of the coefficient matrix. The efficiency of the proposed numerical scheme is demonstrated by computing flow and heat transfer results for natural convection in a cubic cavity for the Rayleigh number range of $10^{3}-10^{6}$, as discussed in the following sections.

\section{GENERALIZED DIFFERENTIAL QUADRATURE METHOD}

The method of GDQ replaces a given partial space derivative of a function $f(x)$ by a linear weighted sum of the function values at the discrete sample points considered along a coordinate direction, resulting in a set of algebraic equations. Hence the GDQ method can be used to obtain numerical solution of partial differential equations with higher-order accuracy. The details about this method can be obtained elsewhere $[12,20]$. For a function of three variables $f(x, y, z)$, the $p$ th-order derivatives, $q$ th-order derivatives, and $r$ th-order derivatives of the function with 
respect to $x, y$, and $z$ can be obtained as

$$
\begin{aligned}
& f_{x}^{(p)}\left(x_{i}, y_{j}, z_{k}\right)=\sum_{l=1}^{L} A_{i, l}^{(p)} f\left(x_{l}, y_{j}, z_{k}\right) \quad p=1,2, \ldots, L-1 \\
& f_{x}^{(q)}\left(x_{i}, y_{j}, z_{k}\right)=\sum_{m=1}^{M} B_{j, m}^{(q)} f\left(x_{l}, y_{m}, z_{k}\right) \quad q=1,2, \ldots, M-1 \\
& f_{x}^{(r)}\left(x_{i}, y_{j}, z_{k}\right)=\sum_{n=1}^{N} C_{k, n}^{(r)} f\left(x_{i}, y_{j}, z_{n}\right) \quad r=1,2, \ldots, N-1 \\
& \text { for } i=1,2, \ldots, L ; j=1,2, \ldots, M ; k=1,2, \ldots, N
\end{aligned}
$$

where $l, m, n$ represent the indices for the grid points in the $x, y$, and $z$ coordinates, respectively; $L, M, N$ are the number of grid points in the $x, y, z$ directions, respectively; and $A_{i, l}^{(p)}, B_{j, m}^{(q)}, C_{k, n}^{(r)}$ are the weighting coefficients. The first-order weighting coefficients $A_{i, l}^{(1)}, B_{j, m}^{(1)}, C_{k, n}^{(1)}$ can be determined as follows:

$$
\begin{gathered}
A_{i, j}^{(1)}=\frac{L^{(1)}\left(x_{i}\right)}{\left(x_{i}-x_{j}\right) L^{(1)}\left(x_{j}\right)} \quad i, j=1,2, \ldots, L, \text { but } j \neq i \\
B_{i, j}^{(1)}=\frac{M^{(1)}\left(y_{i}\right)}{\left(y_{i}-y_{j}\right) M^{(1)}\left(y_{j}\right)} \quad i, j=1,2, \ldots, M, \text { but } j \neq i \\
C_{i, j}^{(1)}=\frac{N^{(1)}\left(z_{i}\right)}{\left(z_{i}-z_{j}\right) N^{(1)}\left(z_{j}\right)} \quad i, j=1,2, \ldots, N, \text { but } j \neq i
\end{gathered}
$$

in which

$$
\begin{gathered}
L^{(1)}\left(x_{i}\right)=\prod_{j=1, j \neq i}^{L}\left(x_{i}-x_{j}\right) \quad M^{(1)}\left(y_{i}\right)=\prod_{j=1, j \neq i}^{M}\left(y_{i}-y_{j}\right) \\
N^{(1)}\left(z_{i}\right)=\prod_{j=1, j \neq i}^{N}\left(z_{i}-z_{j}\right)
\end{gathered}
$$

Similarly, the weighting coefficients for the second- and higher-order derivatives can be obtained as

$A_{i, j}^{(p)}=p\left(A_{i, i}^{(p-1)} A_{i, j}^{(1)}-\frac{A_{i, j}^{(p-1)}}{x_{i}-x_{j}}\right) \quad$ for $i, j=1,2, \ldots, L$, but $j \neq i, l=2,3, \ldots, L-1$ 
$B_{i, j}^{(q)}=q\left(B_{i, i}^{(q-1)} B_{i, j}^{(1)}-\frac{B_{i, j}^{(q-1)}}{y_{i}-y_{j}}\right) \quad$ for $i, j=1,2, \ldots, M$, but $j \neq i, m=2,3, \ldots, M-1$

$C_{i, j}^{(r)}=r\left(C_{i, i}^{(r-1)} C_{i, j}^{(1)}-\frac{C_{i, j}^{(r-1)}}{z_{i}-z_{j}}\right) \quad$ for $i, j=1,2, \ldots, N$, but $j \neq i, n=2,3, \ldots, N-1$

When $j=i$, the weighting coefficients are written as

$$
\begin{aligned}
& A_{i, i}^{(p)}=-\sum_{j=1, j \neq i}^{L} A_{i, j}^{(p)} \quad i=1,2, \ldots, L, p=1,2, \ldots, L-1 \\
& B_{i, i}^{(q)}=-\sum_{j=1, j \neq i}^{M} B_{i, j}^{(q)} \quad i=1,2, \ldots, M, q=1,2, \ldots, M-1 \\
& C_{i, i}^{(r)}=-\sum_{j=1, j \neq i}^{N} C_{i, j}^{(r)} \quad i=1,2, \ldots, N, r=1,2, \ldots, N-1
\end{aligned}
$$

It should be noted from the above equations that the weighting coefficients of the second- and higher-order derivatives can be computed from the first-order derivatives themselves.

\section{GOVERNING EQUATIONS}

The governing equations for natural convection can be described by the incompressible Navier-Stokes equations and the energy equation. Assuming the Boussinesq approximation, the primitive-variable forms of these equations can be written in dimensional form as follows.

Continuity equation:

$$
\nabla \cdot \vec{u}^{*}=0
$$

Momentum equations:

$$
\frac{\partial \vec{u}^{*}}{\partial t^{*}}+\left(\vec{u}^{*} \cdot \nabla\right) \vec{u}^{*}=-\frac{1}{\rho} \nabla P^{*}+v\left(\nabla^{2} \vec{u}^{*}\right)+g \beta\left(T^{*}-T_{\alpha}\right) \vec{k}
$$

Energy equation:

$$
\frac{\partial T^{*}}{\partial t^{*}}+\vec{u}^{*} \cdot\left(\nabla T^{*}\right)=\alpha \nabla^{2} T^{*}
$$

on a Cartesian coordinate frame in which $x-y$ represents the horizontal plane and $z$ refers to the vertical direction. In the velocity-vorticity form of the Navier-Stokes 
equations, the vorticity vector is defined as

$$
\vec{\omega}^{*}=\nabla \times \vec{u}^{*}
$$

By taking the curl on both sides of Eq. (7) and using the vorticity definition, the momentum conservation equation (7) can be rewritten as

$$
\frac{\partial \vec{\omega}^{*}}{\partial t^{*}}+\vec{u}^{*} \cdot \nabla \vec{\omega}^{*}=\vec{\omega}^{*} \cdot \nabla \vec{u}^{*}+v \nabla^{2} \vec{\omega}^{*}+\nabla \times\left[g \beta\left(T^{*}-T_{\alpha}\right)\right] \vec{k}
$$

where $\vec{u}^{*}=\left(u^{*}, v^{*}, w^{*}\right)$ and $\vec{\omega}^{*}=\left(\xi^{*}, \eta^{*}, \varsigma^{*}\right)$ are the velocity and the vorticity vectors in dimensional form representing the components in the $x, y$, and $z$ directions, respectively; $\vec{k}$ is the unit vector in the $z$ direction; $T^{*}=$ dimensional form of temperature; and $T_{\alpha}$ is the reference temperature. The vorticity transport equation (10) is a single vector equation with two unknown vectors, $\vec{u}^{*}$ and $\vec{\omega}^{*}$. A second vector equation can be obtained by taking curl of the vorticity definition given by Eq. (9). After using the vorticity definition and the continuity equation, the second vector equation is obtained as

$$
\nabla^{2} \vec{u}^{*}=-\nabla \times \vec{\omega}^{*}
$$

The above equation is the velocity Poisson equation, which expresses the kinematic relationship between the velocity vector and the vorticity vector. By standard nondimensionalization of Eq. (10), the vorticity transport equation in nondimensional form can be written as

$$
\frac{\partial \vec{\omega}}{\partial t}+\vec{u} \cdot \nabla \vec{\omega}=\vec{\omega} \cdot \nabla \vec{u}+\operatorname{Pr} \nabla^{2} \vec{\omega}+\operatorname{Ra} \operatorname{Pr} \nabla \times(T) \vec{k}
$$

The nondimensional parameters are defined as Prandtl number $\operatorname{Pr}=v / \alpha$ and Rayleigh number $\mathrm{Ra}=g \beta \Delta T L^{3} / \alpha v$.

Similarly the nondimensional form of the velocity Poisson equation (11) can be rewritten as

$$
\nabla^{2} \vec{u}=-\nabla \times \vec{\omega}
$$

Then the energy equation (8) can be written in nondimensional form as

$$
\frac{\partial T}{\partial t}+\vec{u} \cdot(\nabla T)=\nabla^{2} T
$$

Equations (12)-(14) are the final form of the governing equations that characterize the flow and heat transfer during a natural-convection process. These equations have to be solved in a computational domain $\Omega$ which is enclosed by a solid boundary $\Gamma$. For the case of natural convection in a differentially heated cubic cavity, no-slip velocity boundary conditions are assumed on all the boundary walls. The Dirichlet boundary conditions for velocity on all the walls can be imposed as

$$
\vec{u}=\vec{u}_{b}=0
$$


The boundary conditions for the vorticity transport equations are computed from its definition given by Eq. (9). The energy equation is solved by assuming Dirichlet temperature boundary conditions equal to 0.5 and -0.5 , respectively, on the left and the right walls of the cavity. Other walls of the cavity are assumed to be adiabatic for heat transport. The Dirichlet and Neumann boundary conditions for the energy equation can be written as

$$
\begin{gathered}
T=T_{b} \\
\frac{\partial T}{\partial y}=0 \quad \frac{\partial T}{\partial z}=0
\end{gathered}
$$

\section{NUMERICAL SOLUTION}

In the classical numerical solution algorithms [5, 10], an iterative solution procedure is adopted to resolve the coupling between Eqs. (12)-(14). This will result in huge computational effort in terms of computer memory and computational time for 3-D problems. In order to achieve a significant saving in computational effort, a coupled solution scheme via a single global matrix for all the field variables is adopted in the present work. Since all the field variables are solved using a coupled algorithm, the boundary vorticity values are computed implicitly, without the need to compute the boundary vorticity values externally using a separate scheme such as the Taylor's series expansion scheme used by Wong and Baker [5]. The coupled algorithm has also enabled the implicit enforcement of the kinematic Poisson equation as well as the coupling of the velocity and the vorticity at the wall. In addition to this, the use of the GDQ method enables the approximation of the vorticity definition at the boundaries with higher-order accuracy. Hence the continuity constraint and the conservation of the solenoidality of vorticity field are easily satisfied. The time derivatives of the vorticity transport equations and energy equation are discretized using a second-order-accurate, three-time-level implicit scheme. For example, the time derivative of a variable $\varphi$ can be approximated as

$$
\frac{\partial \varphi}{\partial t}=\frac{3 \varphi^{t+1}-4 \varphi^{t}+\varphi^{t-1}}{2 \Delta t}
$$

\subsection{Approximation of the Governing Equations Using the GDQ Method}

Application of the GDQ method to spatial discretization of the governing equations results in a set of algebraic equations. The GDQ form of the governing equations can be expressed as follows.

Velocity-Poisson equations in the three coordinate directions:

$$
\sum_{l=1}^{L} A_{i, l}^{(2)} u_{l, j, k}+\sum_{m=1}^{M} B_{j, m}^{(2)} u_{i, m, k}+\sum_{n=1}^{N} C_{k, n}^{(2)} u_{i, j, n}+\sum_{m=1}^{M} B_{j, m}^{(1)} \zeta_{i, m, k}-\sum_{n=1}^{N} C_{k, n}^{(1)} \eta_{i, j, n}=0
$$




$$
\begin{aligned}
& \sum_{l=1}^{L} A_{i, l}^{(2)} v_{l, j, k}+\sum_{m=1}^{M} B_{j, m}^{(2)} v_{i, m, k}+\sum_{n=1}^{N} C_{k, n}^{(2)} v_{i, j, n}-\sum_{l=1}^{L} A_{i, l}^{(1)} \zeta_{l, j, k}+\sum_{n=1}^{N} C_{k, n}^{(1)} \xi_{i, j, n}=0 \\
& \sum_{l=1}^{L} A_{i, l}^{(2)} w_{l, j, k}+\sum_{m=1}^{M} B_{j, m}^{(2)} w_{i, m, k}+\sum_{n=1}^{N} C_{k, n}^{(2)} w_{i, j, n}+\sum_{l=1}^{L} A_{i, l}^{(1)} \eta_{l, j, k}-\sum_{m=1}^{M} B_{j, m}^{(1)} \xi_{i, m, k}=0
\end{aligned}
$$

Vorticity transport equations in the three coordinate directions:

$$
\begin{aligned}
& \left(\frac{3}{2 \Delta t}\right)\left(\xi_{i, j, k}\right)^{t+1}+\left(u_{i, j, k}^{q} \sum_{l=1}^{L} A_{i, l}^{(1)} \xi_{l, j, k}+v_{i, j, k}^{q} \sum_{m=1}^{M} B_{j, m}^{(1)} \xi_{i, m, k}+w_{i, j, k}^{q} \sum_{n=1}^{N} C_{k, n}^{(1)} \xi_{i, j, n}\right)^{t+1} \\
& -\left(\xi_{i, j, k}^{q} \sum_{l=1}^{L} A_{i, l}^{(1)} u_{l, j, k}+\eta_{i, j, k}^{q} \sum_{m=1}^{M} B_{j, m}^{(1)} u_{i, m, k}+\zeta_{i, j, k}^{q} \sum_{n=1}^{N} C_{k, n}^{(1)} u_{i, j, n}\right)^{t+1} \\
& -\operatorname{Pr}\left(\sum_{l=1}^{L} A_{i, l}^{(2)} \xi_{l, j, k}+\sum_{m=1}^{M} B_{j, m}^{(2)} \xi_{i, m, k}+\sum_{n=1}^{N} C_{k, n}^{(2)} \xi_{i, j, n}\right)^{t+1} \\
& -\operatorname{Ra} \operatorname{Pr}\left(\sum_{m=1}^{M} B_{j, m}^{(1)} T_{i, m, k}\right)^{t+1}=\left(\frac{4}{2 \Delta t}\right) \xi_{i j k}^{t}+\left(\frac{-1}{2 \Delta t}\right) \xi_{i j k}^{t-1}
\end{aligned}
$$

$$
\begin{aligned}
& \left(\frac{3}{2 \Delta t}\right)\left(\eta_{i, j, k}\right)^{t+1}+\left(u_{i, j, k}^{q} \sum_{l=1}^{L} A_{i, l}^{(1)} \eta_{l, j, k}+v_{i, j, k}^{q} \sum_{m=1}^{M} B_{j, m}^{(1)} \eta_{i, m, k}+w_{i, j, k}^{q} \sum_{n=1}^{N} C_{k, n}^{(1)} \eta_{i, j, n}\right)^{t+1} \\
& -\left(\xi_{i, j, k}^{q} \sum_{l=1}^{L} A_{i, l}^{(1)} v_{l, j, k}+\eta_{i, j, k}^{q} \sum_{m=1}^{M} B_{j, m}^{(1)} v_{i, m, k}+\zeta_{i, j, k}^{q} \sum_{n=1}^{N} C_{k, n}^{(1)} v_{i, j, n}\right)^{t+1} \\
& \quad-\operatorname{Pr}\left(\sum_{l=1}^{L} A_{i, l}^{(2)} \eta_{l, j, k}+\sum_{m=1}^{M} B_{j, m}^{(2)} \eta_{i, m, k}+\sum_{n=1}^{N} C_{k, n}^{(2)} \eta_{i, j, n}\right)^{t+1} \\
& \quad+\operatorname{Ra} \operatorname{Pr}\left(\sum_{l=1}^{L} A_{i, l}^{(1)} T_{l, j, k}\right)^{t+1}=\left(\frac{4}{2 \Delta t}\right) \eta_{i j k}^{t}+\left(\frac{-1}{2 \Delta t}\right) \eta_{i j k}^{t-1}
\end{aligned}
$$

$$
\begin{aligned}
& \left(\frac{3}{2 \Delta t}\right)\left(\zeta_{i, j, k}\right)^{t+1}+\left(u_{i, j, k}^{q} \sum_{l=1}^{L} A_{i, l}^{(1)} \zeta_{l, j, k}+v_{i, j, k}^{q} \sum_{m=1}^{M} B_{j, m}^{(1)} \zeta_{i, m, k}+w_{i, j, k}^{q} \sum_{n=1}^{N} C_{k, n}^{(1)} \zeta_{i, j, n}\right)^{t+1} \\
& -\left(\xi_{i, j, k}^{q} \sum_{l=1}^{L} A_{i, l}^{(1)} w_{l, j, k}+\eta_{i, j, k}^{q} \sum_{m=1}^{M} B_{j, m}^{(1)} w_{i, m, k}+\zeta_{i, j, k}^{q} \sum_{n=1}^{N} C_{k, n}^{(1)} w_{i, j, n}\right)^{t+1} \\
& \quad-\operatorname{Pr}\left(\sum_{l=1}^{L} A_{i, l}^{(2)} \zeta_{l, j, k}+\sum_{m=1}^{M} B_{j, m}^{(2)} \zeta_{i, m, k}+\sum_{n=1}^{N} C_{k, n}^{(2)} \zeta_{i, j, n}\right)^{t+1}=\left(\frac{4}{2 \Delta t}\right) \zeta_{i j k}^{t}+\left(\frac{-1}{2 \Delta t}\right) \zeta_{i j k}^{t-1}
\end{aligned}
$$


Energy equation:

$$
\begin{gathered}
\left(\frac{3}{2 \Delta t}\right)\left(T_{i, j, k}\right)^{t+1}+\left(u_{i, j, k}^{q} \sum_{l=1}^{L} A_{i, l}^{(1)} T_{l, j, k}+v_{i, j, k}^{q} \sum_{m=1}^{M} B_{j, m}^{(1)} T_{i, m, k}+w_{i, j, k}^{q} \sum_{n=1}^{N} C_{k, n}^{(1)} T_{i, j, n}\right)^{t+1} \\
-\left(\sum_{l=1}^{L} A_{i, l}^{(2)} T_{l, j, k}+\sum_{m=1}^{M} B_{j, m}^{(2)} T_{i, m, k}+\sum_{n=1}^{N} C_{k, n}^{(2)} T_{i, j, n}\right)^{t+1}=\left(\frac{4}{2 \Delta t}\right) T_{i j k}^{t}+\left(\frac{-1}{2 \Delta t}\right) T_{i j k}^{t-1}
\end{gathered}
$$

Combining Eqs. (17)-(19), all the seven field variables can be represented by means of a single global matrix as

$$
\left[\begin{array}{ccccccc}
A & 0 & 0 & 0 & -D & C & 0 \\
0 & A & 0 & D & 0 & -B & 0 \\
0 & 0 & A & -C & B & 0 & 0 \\
E & 0 & 0 & F & 0 & 0 & G \\
0 & E & 0 & 0 & F & 0 & H \\
0 & 0 & E & 0 & 0 & F & 0 \\
0 & 0 & 0 & 0 & 0 & 0 & I
\end{array}\right]\left\{\begin{array}{c}
u \\
v \\
w \\
\xi \\
\eta \\
\zeta \\
T
\end{array}\right\}=\left\{\begin{array}{c}
f_{u} \\
f_{v} \\
f_{w} \\
f_{\xi} \\
f_{\eta} \\
f_{\zeta} \\
f_{T}
\end{array}\right\}
$$

where $A, B, C, D, E, F, G$, and $H$ are the influence matrices which represent the various differential operators that appear in the GDQ approximation of Eqs. (17)-(19), $u, v, w, \xi, \eta, \zeta$, and $T$ are the vectors representing the unknown field variables, and $f_{u}, f_{v}, f_{w}, f_{\xi}, f_{\eta}, f_{\zeta}, f_{T}$ are the load vectors. The influence matrices and the load vectors are computed as

$$
\begin{aligned}
& {[A]=} \sum_{l=1}^{L} A_{i, l}^{(2)}+\sum_{m=1}^{M} B_{j, m}^{(2)}+\sum_{n=1}^{N} C_{k, n}^{(2)} \\
& {[B]=} \sum_{l=1}^{L} A_{i, l}^{(1)} \quad[C]=\sum_{m=1}^{M} B_{j, m}^{(1)} \quad[D]=\sum_{n=1}^{N} C_{k, n}^{(1)} \\
& {[E]=-\left(\xi_{i, j, k}^{q} \sum_{l=1}^{L} A_{i, l}^{(1)}+\eta_{i, j, k}^{q} \sum_{m=1}^{M} B_{j, m}^{(1)}+\zeta_{i, j, k}^{q} \sum_{n=1}^{N} C_{k, n}^{(1)}\right) } \\
& {[F]=\frac{3}{2 \Delta t}+\left(u_{i, j, k}^{q} \sum_{l=1}^{L} A_{i, l}^{(1)}+v_{i, j, k}^{q} \sum_{m=1}^{M} B_{j, m}^{(1)}+w_{i, j, k}^{q} \sum_{n=1}^{N} C_{k, n}^{(1)}\right) } \\
& \quad-\operatorname{Pr}\left(\sum_{l=1}^{L} A_{i, l}^{(2)}+\sum_{m=1}^{M} B_{j, m}^{(2)}+\sum_{n=1}^{N} C_{k, n}^{(2)}\right) \\
& {[G]=-\operatorname{Ra} \operatorname{Pr} \sum_{m=1}^{M} B_{j, m}^{(1)}[H]=\operatorname{Ra} \operatorname{Pr} \sum_{l=1}^{L} A_{i, l}^{(1)} } \\
& {[I]=} \frac{3}{2 \Delta t}+\left(u_{i, j, k}^{q} \sum_{l=1}^{L} A_{i, l}^{(1)}+v_{i, j, k}^{q} \sum_{m=1}^{M} B_{j, m}^{(1)}+w_{i, j, k}^{q} \sum_{n=1}^{N} C_{k, n}^{(1)}\right) \\
&-\left(\sum_{l=1}^{L} A_{i, l}^{(2)}+\sum_{m=1}^{M} B_{j, m}^{(2)}+\sum_{n=1}^{N} C_{k, n}^{(2)}\right)
\end{aligned}
$$




$$
\begin{array}{ll}
{\left[f_{u}\right]=0 \quad\left[f_{v}\right]=0 \quad\left[f_{w}\right]=0} \\
{\left[f_{\xi}\right]=\frac{4}{2 \Delta t} \xi^{t}+\frac{-1}{2 \Delta t} \xi^{t-1}} & {\left[f_{\eta}\right]=\frac{4}{2 \Delta t} \eta^{t}+\frac{-1}{2 \Delta t} \eta^{t-1}} \\
{\left[f_{\zeta}\right]=\frac{4}{2 \Delta t} \zeta^{t}+\frac{-1}{2 \Delta t} \zeta^{t-1}} & {\left[f_{T}\right]=\frac{4}{2 \Delta t}(T)^{t}+\frac{-1}{2 \Delta t}(T)^{t-1}}
\end{array}
$$

where $q$ is the iterative number index and $t$ is the time-level index.

\subsection{Determination of Vorticity Boundary Conditions}

The vorticity boundary conditions for all the three vorticity transport equations in the principal coordinate directions are computed using the vorticity definition given by Eq. (9). The velocity gradients used in this definition can be computed with a higher-order accuracy using the first-order weighting coefficients $A_{i, l}^{(1)}, B_{j, m}^{(1)}, C_{k, n}^{(1)}$. By applying the GDQ approximation to the vorticity definition given by Eq. (9), the three vorticity components on a boundary can be expressed as

$$
\begin{aligned}
& \xi_{i, j, k}-\sum_{m=1}^{M} B_{j, m}^{(1)} w_{i, m, k}+\sum_{n=1}^{N} C_{k, n}^{(1)} v_{i, j, n}=0 \\
& \eta_{i, j, k}+\sum_{l=1}^{L} A_{i, l}^{(1)} w_{l, j, k}-\sum_{n=1}^{N} C_{k, n}^{(1)} u_{i, j, n}=0 \\
& \zeta_{i, j, k}-\sum_{l=1}^{L} A_{i, l}^{(1)} v_{l, j, k}+\sum_{m=1}^{M} B_{j, m}^{(1)} u_{i, m, k}=0
\end{aligned}
$$

The Dirichlet boundary conditions for the temperature are expressed as

$$
\begin{array}{rl}
T_{L, j, k}=0.5 & j=1, \ldots, M, \quad k=1, \ldots, N \\
T_{1, j, k}=-0.5 \quad j=1, \ldots, M, \quad k=1, \ldots, N
\end{array}
$$

The adiabatic boundary conditions can be achieved by computing the normal derivatives of the temperature at the adiabatic walls and equating them to zero. Hence the GDQ forms of the adiabatic boundary conditions can be represented by the following expressions [20]:

$$
\begin{aligned}
T_{i, 1, k} & =\frac{1}{B_{1,1}^{(1)} B_{M, M}^{(1)}-B_{1, M}^{(1)} B_{M, 1}^{(1)}}\left[\sum_{m=2}^{M-1}\left(B_{1, M}^{(1)} B_{M, m}^{(1)}-B_{M, M}^{(1)} B_{1, m}^{(1)}\right) T_{i, m, k}\right] \\
T_{i, M, k} & =\frac{1}{B_{1,1}^{(1)} B_{M, M}^{(1)}-B_{1, M}^{(1)} B_{M, 1}^{(1)}}\left[\sum_{m=2}^{M-1}\left(B_{M, 1}^{(1)} B_{1, m}^{(1)}-B_{1,1}^{(1)} B_{M, m}^{(1)}\right) T_{i, m, k}\right] \\
T_{i, j, 1} & =\frac{1}{C_{1,1}^{(1)} C_{N, N}^{(1)}-C_{1, N}^{(1)} C_{N, 1}^{(1)}}\left[\sum_{n=2}^{N-1}\left(C_{1, N}^{(1)} C_{N, n}^{(1)}-C_{N, N}^{(1)} C_{1, n}^{(1)}\right) T_{i, j, n}\right]
\end{aligned}
$$




$$
T_{i, j, N}=\frac{1}{C_{1,1}^{(1)} C_{N, N}^{(1)}-C_{1, N}^{(1)} C_{N, 1}^{(1)}}\left[\sum_{n=2}^{N-1}\left(C_{N, 1}^{(1)} C_{1, n}^{(1)}-C_{1,1}^{(1)} C_{N, n}^{(1)}\right) T_{i, j, n}\right]
$$

Equations (23a)-(23d) also involve the implicit scheme for the Neumann boundary conditions. The simultaneous equations resulting from the global matrix system of Eq. (20) are solved using a BiCG iterative equation solver. Since the coefficient matrix of the global matrix system is sparse, only the nonzero entries are stored in column storage format. In the present study we employed the following convergence criteria to terminate the iterative process used to resolve the coupling between the field variables at a given time step:

$$
\begin{array}{ll}
\left|\left(u_{q+1}^{t+1}-u_{q}^{t+1}\right) / u_{q}^{t+1}\right| \leq 10^{-6} & \left|\left(v_{q+1}^{t+1}-v_{q}^{t+1}\right) / v_{q}^{t+1}\right| \leq 10^{-6} \\
\left|\left(w_{q+1}^{t+1}-w_{q}^{t+1}\right) / w_{q}^{t+1}\right| \leq 10^{-6} & \left|\left(\xi_{q+1}^{t+1}-\xi_{q}^{t+1}\right) / \xi_{q}^{t+1}\right| \leq 10^{-6} \\
\left|\left(\eta_{q+1}^{t+1}-\eta_{q}^{t+1}\right) / \eta_{q}^{t+1}\right| \leq 10^{-6} & \left|\left(\zeta_{q+1}^{t+1}-\zeta_{q}^{t+1}\right) / \zeta_{q}^{t+1}\right| \leq 10^{-6} \\
\left|\left(T_{q+1}^{t+1}-T_{q}^{t+1}\right) / T_{q}^{t+1}\right| \leq 10^{-6} &
\end{array}
$$

In the successive time step, we used the velocity, vorticity, and temperature components at the previous time step as the initial guess for the next iteration. The computations are carried out until steady-state conditions are reached. The convergence criteria used in the time loop to achieve steady-state conditions are

$$
\begin{array}{lll}
\left|\left(u^{t+1}-u^{t}\right) / u^{t}\right| \leq 10^{-6} & \left|\left(v^{t+1}-v^{t}\right) / v^{t}\right| \leq 10^{-6} & \left|\left(w^{t+1}-w^{t}\right) / w^{t}\right| \leq 10^{-6} \\
\left|\left(\xi^{t+1}-\xi^{t}\right) / \xi^{t}\right| \leq 10^{-6} & \left|\left(\eta^{t+1}-\eta^{t}\right) / \eta^{t}\right| \leq 10^{-6} & \left|\left(\zeta^{t+1}-\zeta^{t}\right) / \zeta^{t}\right| \leq 10^{-6} \\
\left|\left(T^{t+1}-T^{t}\right) / T^{t}\right| \leq 10^{-6} & &
\end{array}
$$

For the GDQ method, the mesh point distribution in the three spatial coordinates is assumed to be the same and is expressed as

$$
\begin{aligned}
& x_{i}=\frac{\cos [\pi /(2 L)]-\cos [(2 i-1) \pi /(2 L)]}{\cos [\pi /(2 L)]-\cos [(2 L-1) \pi /(2 L)]} \quad i=1,2, \ldots, L \\
& y_{j}=\frac{\cos [\pi /(2 M)]-\cos [(2 j-1) \pi /(2 M)]}{\cos [\pi /(2 M)]-\cos [(2 M-1) \pi /(2 M)]} \quad j=1,2, \ldots, M \\
& z_{k}=\frac{\cos [\pi /(2 N)]-\cos [(2 k-1) \pi /(2 N)]}{\cos [\pi /(2 N)]-\cos [(2 N-1) \pi /(2 N)]} \quad k=1,2, \ldots, N
\end{aligned}
$$

where $L, M, N$ are the number of grid points in the $x, y$, and $z$ directions, respectively.

\section{RESULTS AND DISCUSSIONS}

The accuracy of the present numerical procedure is verified by (1) a grid independence study and (2) validating the predicted results with the benchmark 
solutions. Air is assumed to be the working fluid, with $\operatorname{Pr}=0.71$ for the naturalconvection simulation cases.

\subsection{Grid Independence Study}

For the grid independence study, numerical results are obtained for natural convection in a cubic cavity (see Figure 1 ) for $\mathrm{Ra}=10^{3}$ and $10^{4}$. The computational grid was refined successively from $15^{3}$ to $19^{3}$ and $25^{3}$. The following mean and overall Nusselt numbers are considered as the parameters for comparison purposes.

1. The mean Nusselt number throughout the cavity is

$$
\mathrm{Nu}_{\text {mean }}(y)=\left.\int_{0}^{1} \frac{\partial T(y, z)}{\partial x}\right|_{x=-0.5, \text { or } x=0.5} d z
$$

2. The overall Nusselt number on the boundary at $x=-0.5$ or $x=0.5$ is

$$
\mathrm{Nu}_{\text {overall }}=\int_{0}^{1} \mathrm{Nu}_{\text {mean }}(y) d y
$$

The effect of grid refinement on the values of the mean and the overall Nusselt numbers is shown in Table 1 for $\mathrm{Ra}=10^{3}$ and $10^{4}$. As the mesh is refined successively from $15^{3}$ to $19^{3}$ and $25^{3}$, there is a consistent improvement in the accuracy of the predicted values of the Nusselt numbers for both values of the Rayleigh number, with no difference being observed between the grids $19^{3}$ and $25^{3}$. This indicates that the $25^{3}$ grid can produce grid-independent numerical results and hence will be used throughout this work. The grid independence study is also demonstrated by

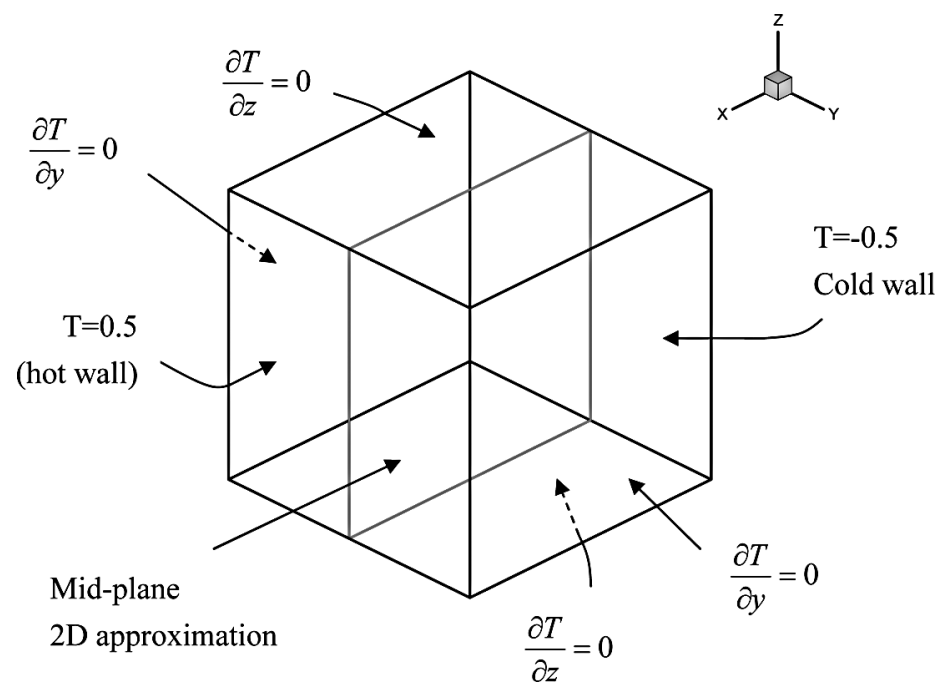

Figure 1. Geometry and boundary conditions for the buoyancy-driven cavity problem. 
Table 1. Grid independence study results for $\mathrm{Ra}=10^{3}$ and $10^{4}$

\begin{tabular}{|c|c|c|c|c|c|c|c|c|}
\hline \multirow{2}{*}{$\begin{array}{l}\text { Ra } \\
\text { Method }\end{array}$} & \multicolumn{4}{|c|}{$10^{3}$} & \multicolumn{4}{|c|}{$10^{4}$} \\
\hline & GDQ & GDQ & GDQ & PSC [15] & GDQ & GDQ & GDQ & PSC [15] \\
\hline Mesh size & $15^{3}$ & $19^{3}$ & $25^{3}$ & $32^{3}$ & $15^{3}$ & $19^{3}$ & $25^{3}$ & $61^{3}$ \\
\hline $\mathrm{Nu}_{\text {overall }}$ & 1.069 & 1.070 & 1.070 & 1.070 & 2.052 & 2.054 & 2.054 & 2.0542 \\
\hline $\mathrm{Nu}_{\text {mean }}(y=0.5)$ & 1.085 & 1.087 & 1.087 & 1.0873 & 2.248 & 2.251 & 2.251 & 2.2505 \\
\hline
\end{tabular}

plotting the temperature profiles along the centerline of the symmetry plane at $y=0.5$. Figures $2 a$ and $2 b$ show the effect of grid refinement on the temperature profiles for $\mathrm{Ra}=10^{3}$ and $10^{4}$, respectively. The temperature profiles of all three meshes almost coincide with each other for both $\mathrm{Ra}=10^{3}$ and $10^{4}$. The effect of grid refinement on the velocity profile $u-z$ along the vertical symmetric central line is shown in Figures $3 a-1$ and $3 a-2$ for $\mathrm{Ra}=10^{3}$ and $10^{4}$, respectively. Similarly, Figures $3 b-1$ and $3 b-2$ represent the $x-w$ plots along the horizontal symmetric central line for $\mathrm{Ra}=10^{3}$ and $10^{4}$, respectively. The exact coincidence of the above velocity profiles for all three meshes indicates that the present numerical algorithm based on the velocityvorticity formulation and the GDQ method predicts the temperature and flow results consistent with grid refinement.

\subsection{Validation of Numerical Results}

For the case of a natural-convection problem, the effect of Rayleigh number on the convective heat transfer between the end walls is evaluated in terms of the mean and the overall Nusselt number for $\mathrm{Ra}=10^{3}, 10^{4}, 10^{5}$, and $10^{6}$. In order to validate the present numerical algorithm, the predicted results are compared with the results obtained by three different numerical schemes: (1) the pseudo-spectral Chebyshev algorithm (Tric et al., 2000), (2) the lattice Boltzmann model (Peng et al., 2003), and (3) the finite-difference method (Fusegi et al., 1991). Table 2 shows the comparison of the present results with the results of the above three numerical schemes for $\mathrm{Ra}=10^{3}$ and $10^{4}$. For both Rayleigh numbers, the present results are in close agreement with the results of the above three numerical schemes, which adopted grids finer than $25^{3}$, used in the present method. Table 3 shows a similar comparison for $\mathrm{Ra}=10^{5}$ and $10^{6}$. Even for higher Rayleigh number values, the present results obtained using $25^{3}$ grid are closer to the results of Tric et al. [15], who used a grid size of $81^{3}$. A close look at the tabular values indicates that the present predictions are in better agreement with the results of Tric et al. [15] compared to the results of the other two numerical schemes [16, 17], obtained using finer meshes. For all four values of Rayleigh number considered in the present analysis, the average and overall Nusselt numbers on the isothermal wall have been predicted with less than $0.4 \%$ error in comparison to the benchmark results of Tric et al. [15]. Though Wong and Baker [5] also solved the velocity-vorticity equations to study natural convection in a cubic cavity, they had to use a fine mesh of size $48^{3}$ in order to compute the vorticity boundary values using a second-order-accurate Taylor's series scheme, since they employed the finite-element method. Moreover, the numerical schemes used in 


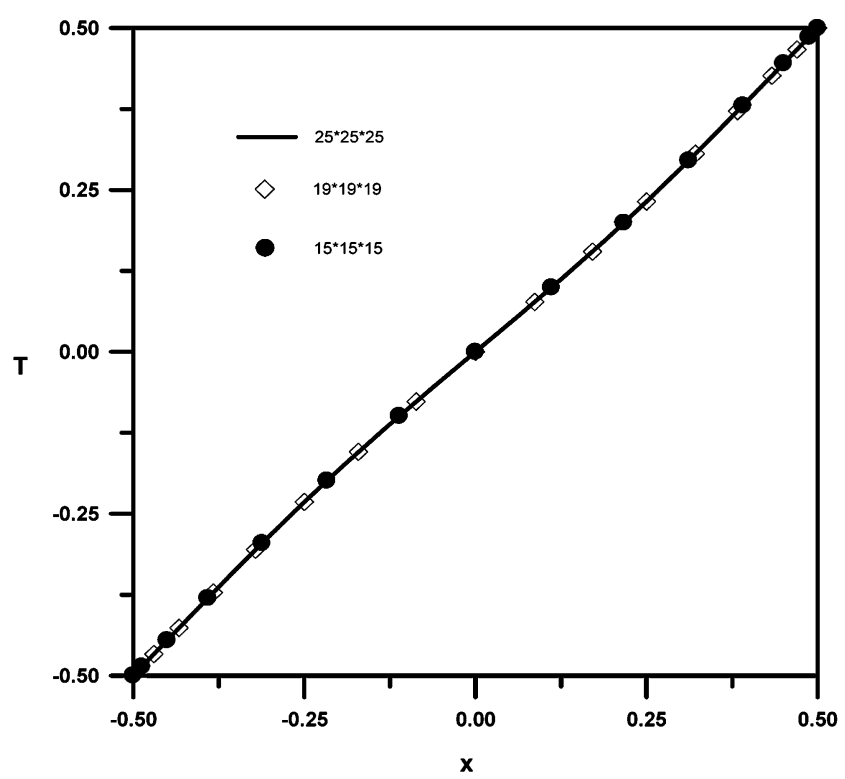

(a)

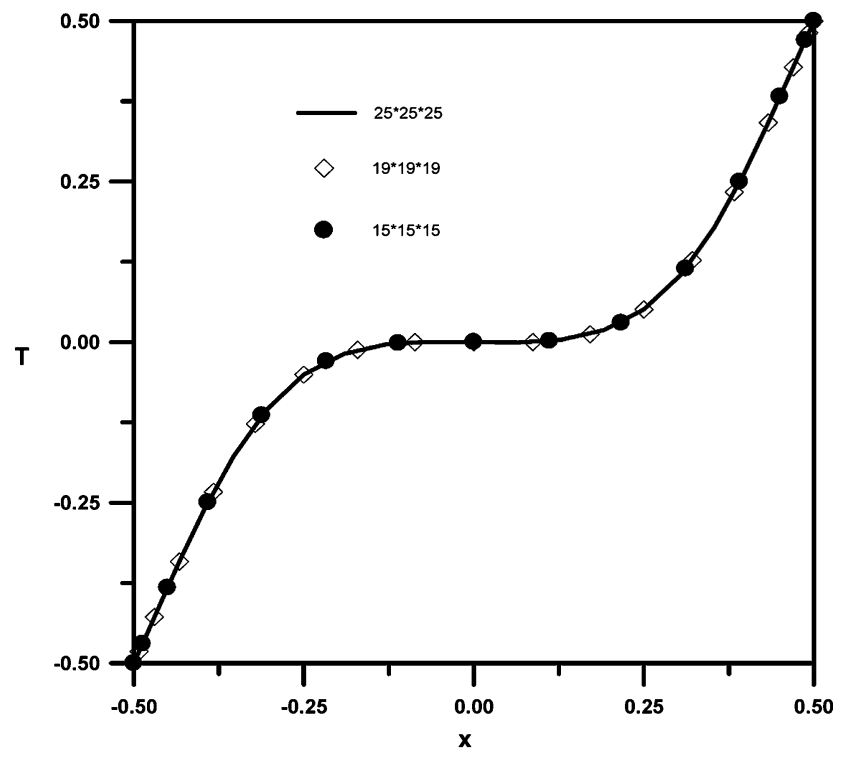

(b)

Figure 2. Temperature profiles in the centerline of the symmetry plane at $y=0.5$ for $(a) \operatorname{Ra}=10^{3}$, (b) $\mathrm{Ra}=10^{4}$.

[5, 15-17] were implemented on high-speed computers such as Cray computers. Using the present numerical algorithm, the benchmark results could be achieved using a coarse mesh on a Pentium IV personal computer. The reason is that the 


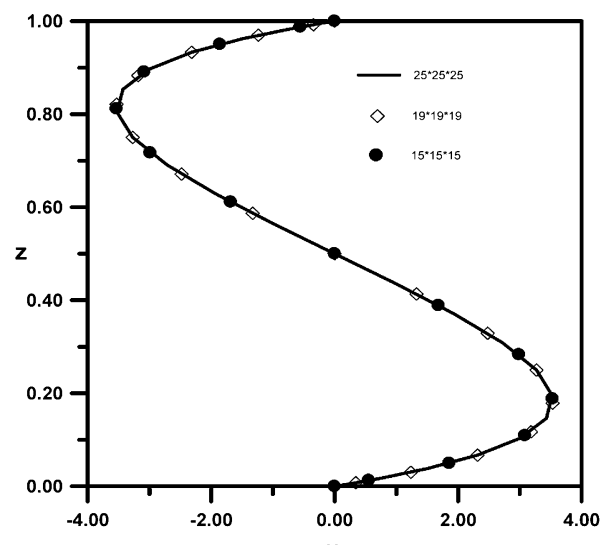

(a-1)

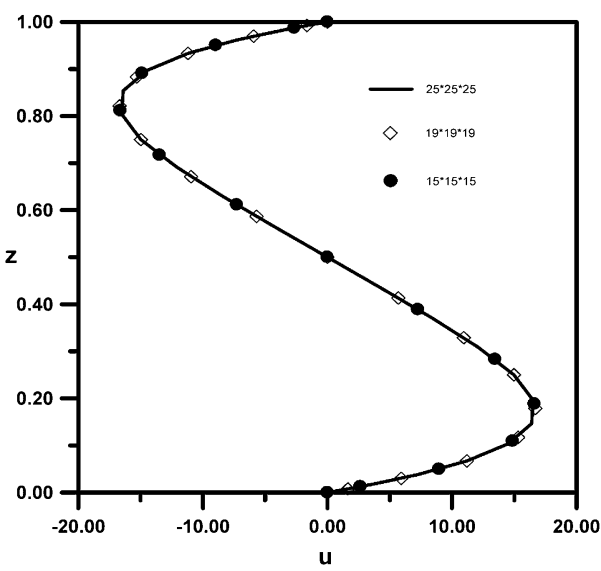

(b-1)

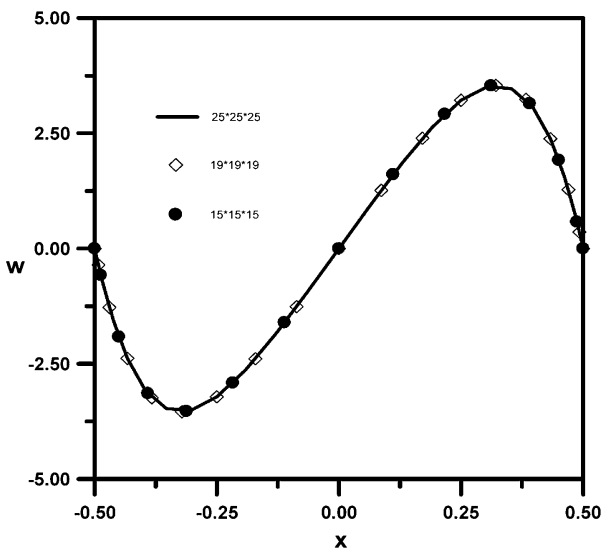

$(\mathrm{a}-2)$

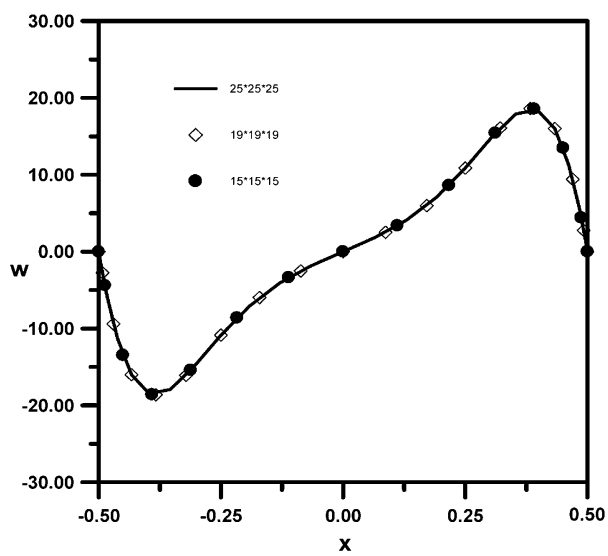

$(b-2)$

Figure 3. Velocity profiles along the centerline in the symmetry plane at $y=0.5$ for $(a) \operatorname{Ra}=10^{3},(b)$ $\mathrm{Ra}=10^{4}$.

Table 2. Validation results for $\mathrm{Ra}=10^{3}$ and $10^{4}$ : Comparison of Nusselt number at the isothermal wall, $\mathrm{x}=-0.5$

\begin{tabular}{|c|c|c|c|c|c|c|c|c|}
\hline \multirow{2}{*}{$\begin{array}{l}\text { Ra } \\
\text { Method }\end{array}$} & \multicolumn{4}{|c|}{$10^{3}$} & \multicolumn{4}{|c|}{$10^{4}$} \\
\hline & GDQ & PSC [15] & LBM [16] & FDM [17] & GDQ & PSC [15] & LBM [16] & FDM [17] \\
\hline Mesh size & $25^{3}$ & $61^{3}$ & $81^{3}$ & $32^{3}$ & $25^{3}$ & $61^{3}$ & $61 \times 45 \times 45$ & $62^{3}$ \\
\hline $\mathrm{Nu}_{\text {overall }}$ & 1.070 & 1.070 & 1.075 & 1.085 & 2.054 & 2.0542 & 2.085 & 2.100 \\
\hline $\mathrm{Nu}_{\text {mean }}(y=0.5)$ & 1.087 & 1.0873 & 1.097 & 1.105 & 2.251 & 2.2505 & 2.304 & 2.302 \\
\hline
\end{tabular}


Table 3. Validation results for $\mathrm{Ra}=10^{5}$ and $10^{6}$ : Comparison of Nusselt number at the isothermal wall, $x=-0.5$

\begin{tabular}{|c|c|c|c|c|c|c|c|c|}
\hline \multirow{2}{*}{$\begin{array}{l}\text { Ra } \\
\text { Method }\end{array}$} & \multicolumn{4}{|c|}{$10^{5}$} & \multicolumn{4}{|c|}{$10^{6}$} \\
\hline & GDQ & PSC [15] & LBM [16] & FDM [17] & GDQ & PSC [15] & LBM [16] & FDM [17] \\
\hline Mesh size & $25^{3}$ & $81^{3}$ & $91 \times 65 \times 65$ & $62^{3}$ & $25^{3}$ & $81^{3}$ & $\mathrm{~N} / \mathrm{A}$ & $62^{3}$ \\
\hline $\mathrm{Nu}_{\text {overall }}$ & 4.335 & 4.3370 & 4.378 & 4.361 & 8.666 & 8.6407 & $\mathrm{~N} / \mathrm{A}$ & 8.770 \\
\hline $\mathrm{Nu}_{\text {mean }}(y=0.5)$ & 4.610 & 4.6127 & 4.658 & 4.646 & 8.91 & 8.8771 & $\mathrm{~N} / \mathrm{A}$ & 9.012 \\
\hline
\end{tabular}

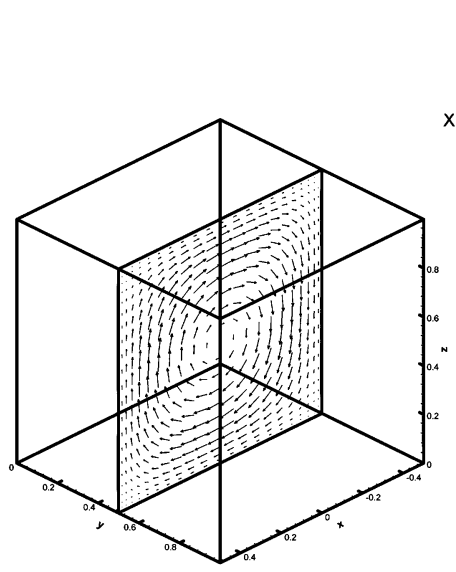

(a)

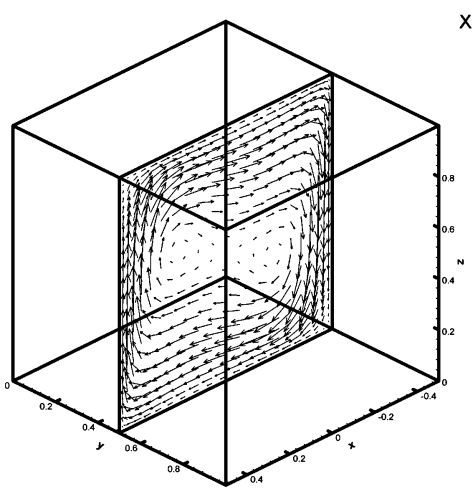

(c)
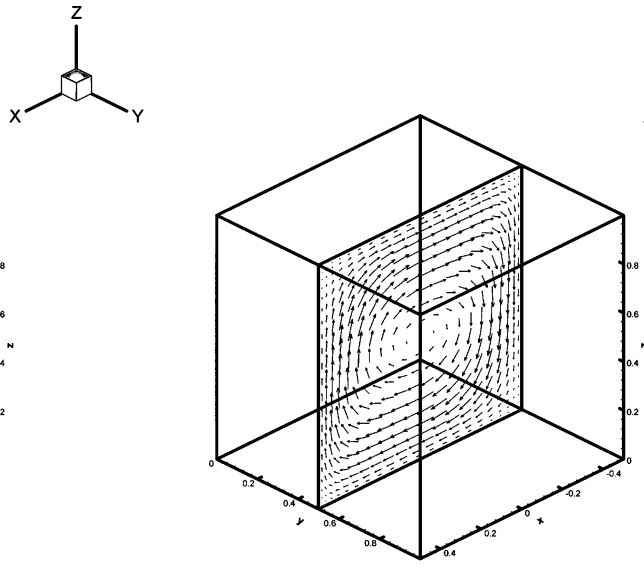

(b)
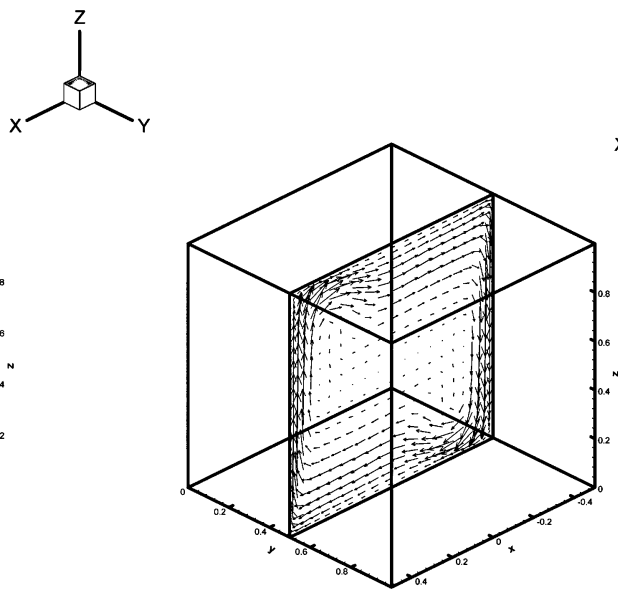

(d)

Figure 4. Velocity vectors at $y=0.5$ plane for $(a) \mathrm{Ra}=10^{3},(b) \mathrm{Ra}=10^{4},(c) \mathrm{Ra}=10^{5}$, (d) $\mathrm{Ra}=10^{6}$. 


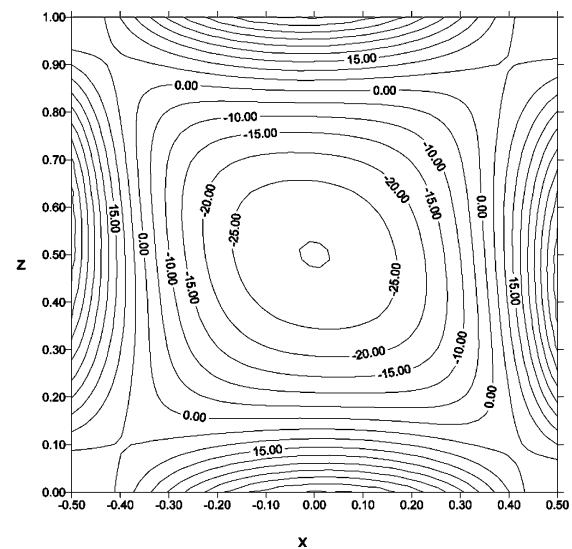

(a)

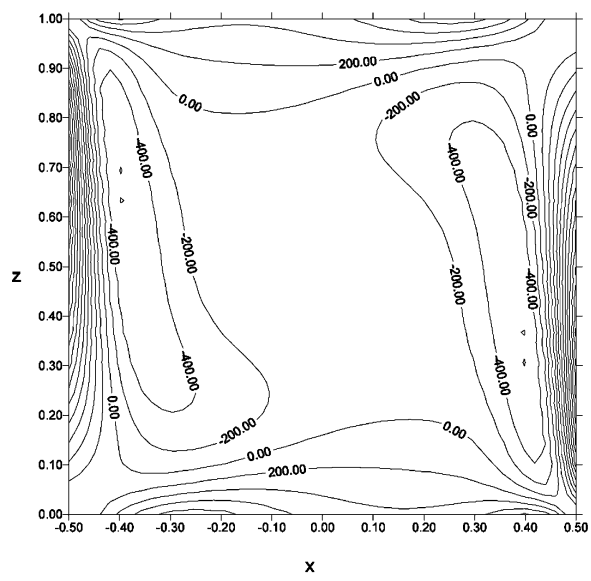

(c)

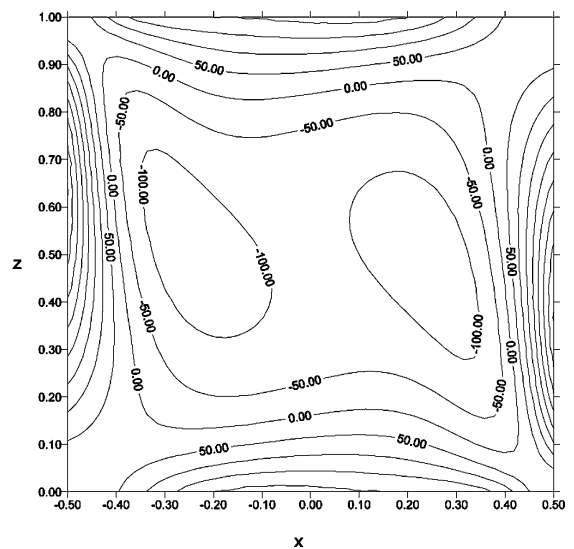

(b)

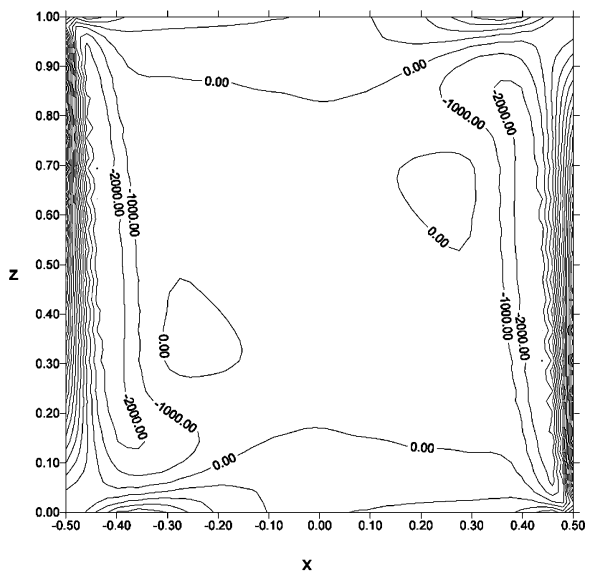

(d)

Figure 5. Vorticity contours at $y=0.5$ plane for $(a) \mathrm{Ra}=10^{3}$, (b) $\mathrm{Ra}=10^{4},(c) \mathrm{Ra}=10^{5},(d) \mathrm{Ra}=10^{6}$.

GDQ method makes use of all the grid points in a given coordinate direction while approximating a differential with respect to that coordinate direction. Hence, while computing the vorticity values at the boundary, there is no need to use very fine mesh near the boundary as required in the finite-element scheme [5].

\subsection{Results on Flow and Thermal Characteristics}

The ability of the present coupled numerical scheme is further demonstrated by plotting the velocity, vorticity, and temperature contours on various symmetric mid-planes along the principal axes of the cubic cavity. Figures $4 a-4 d$ show the velocity vector distributions on the $x-z$ plane at $y=0.5$ for $\mathrm{Ra}=10^{3}, 10^{4}, 10^{5}$, and $10^{6}$. As the Rayleigh number increases, the flow is characterized by a combination of an 


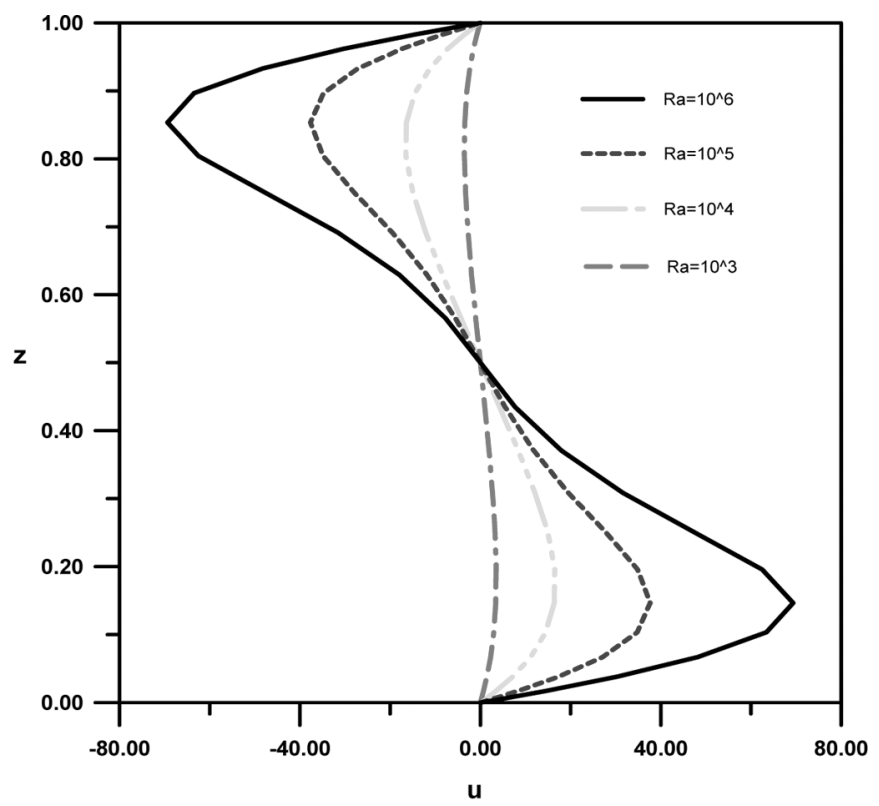

(a)

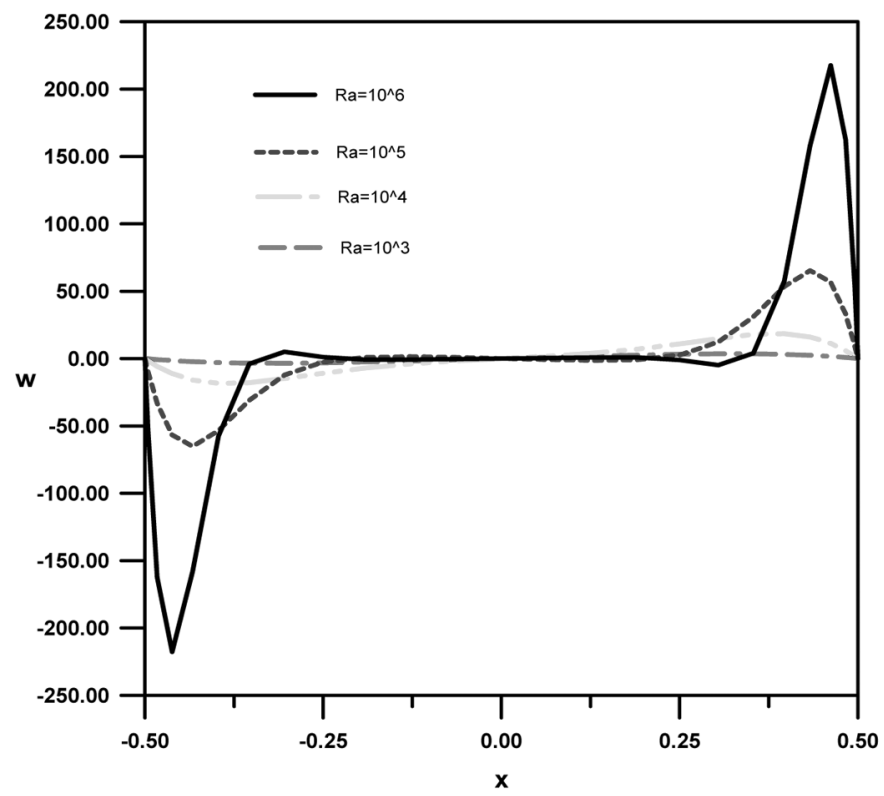

(b)

Figure 6. Centerline velocity profiles of the symmetry plane for $10^{3} \leq \mathrm{Ra} \leq 10^{6}$. 


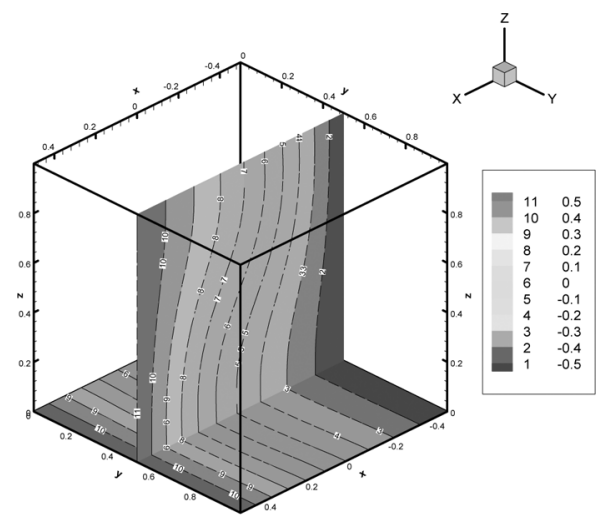

(a)

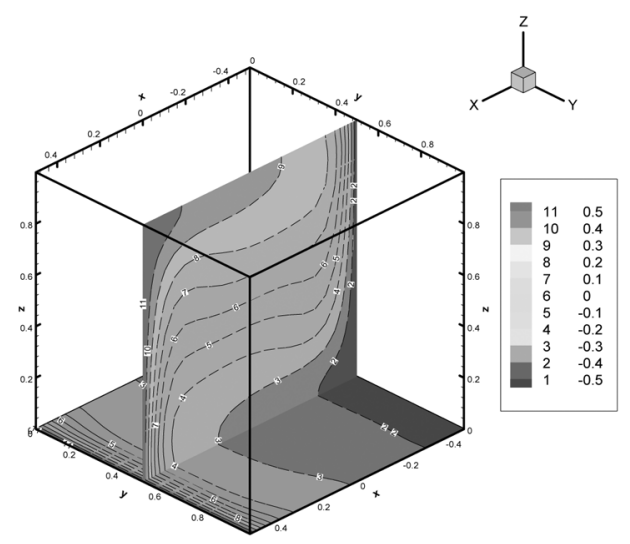

(c)

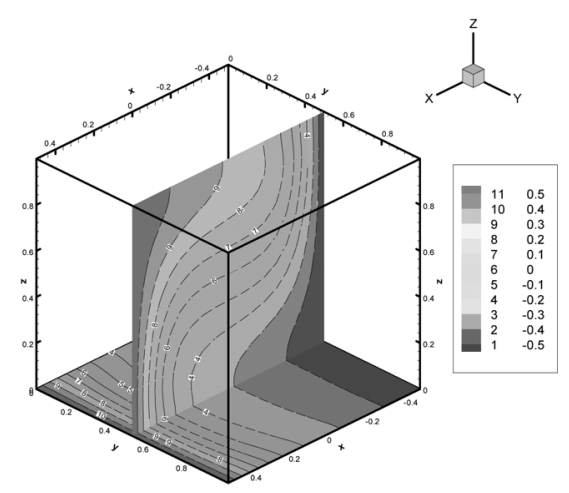

(b)

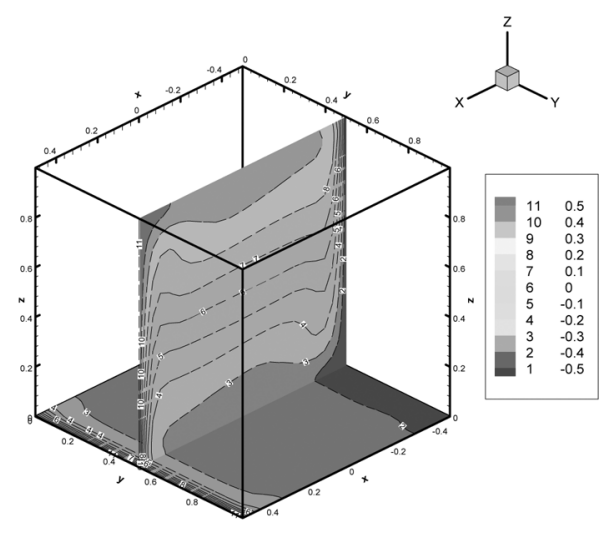

(d)

Figure 7. Contour maps of temperature at $y=0.5$ plane for $(a) \mathrm{Ra}=10^{3},(b) \mathrm{Ra}=10^{4}$, (c) $\mathrm{Ra}=10^{5}$, (d) $\mathrm{Ra}=10^{6}$.

almost stagnant interior fluid core and distinct boundary layers near the side walls. Since the vorticity is the primitive variable in the present formulation, the vortical flow patterns due to the natural convection are obtained directly. The vorticity contour distributions on the $x-z$ plane at $y=0.5$ are shown in Figures $5 a-5 d$ for $\mathrm{Ra}=10^{3}, 10^{4}, 10^{5}$, and $10^{6}$. The vorticity values near the isothermal walls increase with increase in the value of Rayleigh number, resulting in a strong circulatory motion. Higher values of vorticity on the isothermal walls indicate the intense convection effect on the fluid structure. The above constant vorticity contours clearly demonstrate again the existence of a near-stagnant interior core along with the distinct boundary layers near the end walls.

Figures $6 a$ and $6 b$ show the variations of $u$ velocity along the vertical centerline and $w$ velocity along the horizontal centerline at the $y=0.5$ plane for $\operatorname{Ra}=10^{3}, 10^{4}$, $10^{5}$, and $10^{6}$. The peak values of the horizontal and the vertical velocities increase due to the intensified convective activities with increase in Rayleigh number. The 


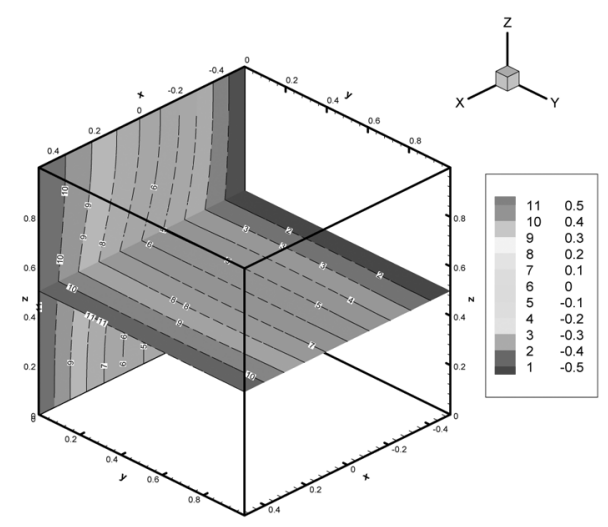

(a)

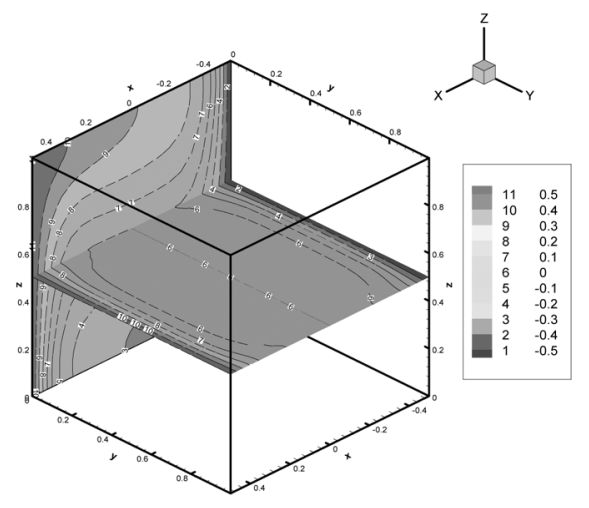

(c)

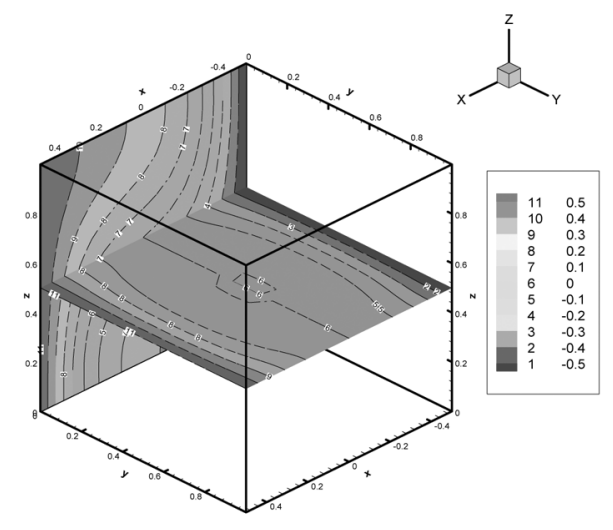

(b)

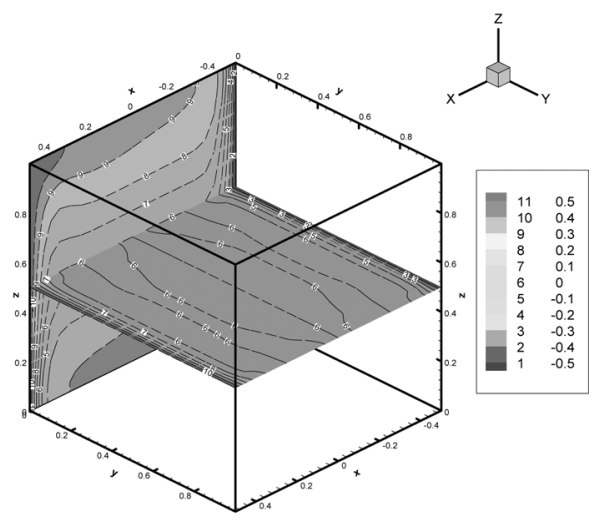

(d)

Figure 8. Contour map of temperature $T$ at $z=0.5$ for $\operatorname{Pr}=0.71$ and Rayleigh numbers $(a) \operatorname{Ra}=10^{3},(b)$ $\mathrm{Ra}=10^{4},(c) \mathrm{Ra}=10^{5},\left(\right.$ d) $\mathrm{Ra}=10^{6}$.

steep rise in the $w$-velocity gradient at the points on the hot and the cold walls also confirm the increased convective activity at higher Rayleigh number values as observed in Figure $6 b$. The $u-z$ and $x-w$ velocity profiles predicted in the present work are in qualitative agreement with the results of Wong and Baker [5].

The temperature contours on the $x-z$ plane at $y=0.5$ are shown in Figure 7 for $\mathrm{Ra}=10^{3}, 10^{4}, 10^{5}$, and $10^{6}$. With increase in Rayleigh number, a high degree of convection is observed such that distinct thermal boundary layers start appearing near the isothermal walls. The thickness of the thermal boundary layer decreases as Rayleigh number increases. The 3-D variations of temperature field can be understood by plotting the temperature contours on the $x-y$ plane at $z=0.5$ as shown in Figures $8 a-8 d$ for different Rayleigh numbers. Sharp temperature changes are noted at the left and right edges of the $x-y$ plane due to the increase in the Rayleigh number and are clearly indicated in the above figures in combination with temperature contours on the vertical plane at $y=1$. Figures $9 a-9 d$ represent the temperature 


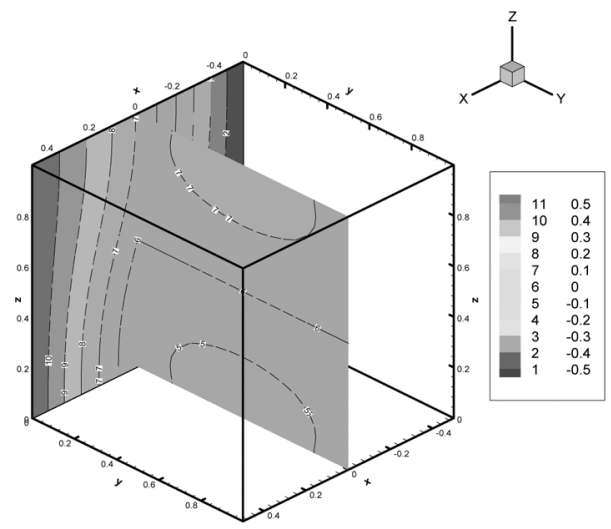

(a)

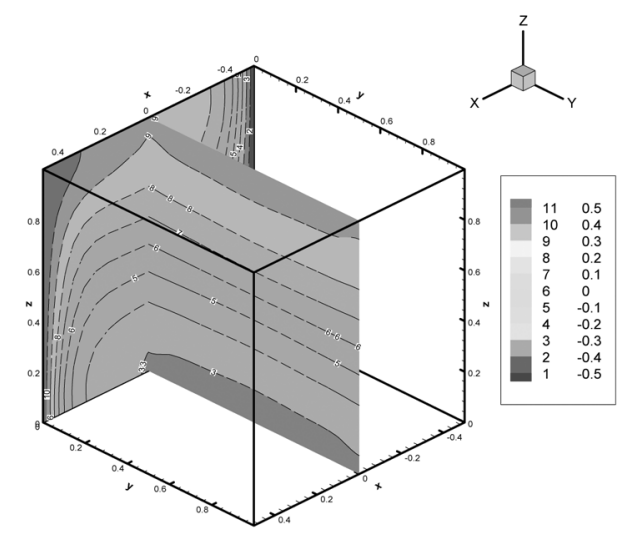

(c)

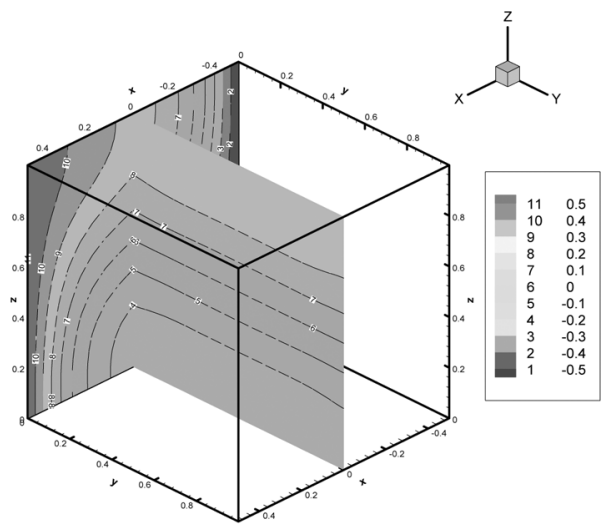

(b)

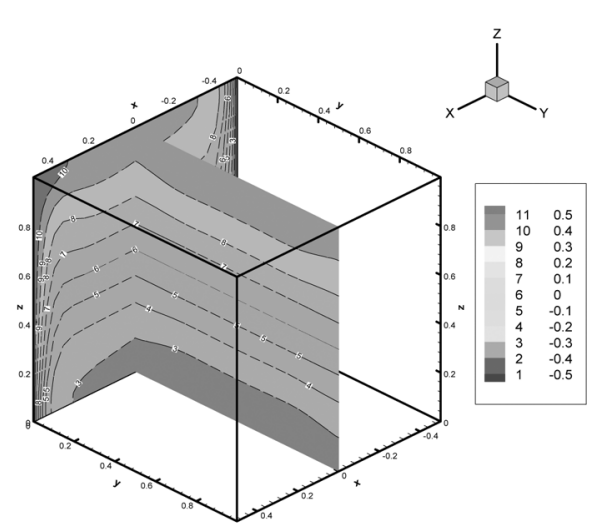

(d)

Figure 9. Contour map of temperature $T$ at $x=0$ for $\operatorname{Pr}=0.71$ and Rayleigh numbers $(a) \operatorname{Ra}=10^{3}$, (b) $\mathrm{Ra}=10^{4},(c) \mathrm{Ra}=10^{5},(d) \mathrm{Ra}=10^{6}$.

contours on the $y-z$ plane at $x=0$ for different Rayleigh numbers. The vertical stratification of the temperature field becomes distinct with increase in Rayleigh number, as indicated in these figures. The accurate predictions of the coincidence of the temperature profiles on the $x=0$ plane with those on the $y=1$ plane demonstrate that the present numerical scheme is capable of predicting the 3-D variation of the temperature field accurately. In order to understand the 3-D convective effect, the variation of the mean Nusselt number along the $y$ direction are plotted in Figures $10 a-10 d$ for $\mathrm{Ra}=10^{3}, 10^{4}, 10^{5}$, and $10^{6}$. As the Rayleigh number increases, the mean Nusselt number increases as the symmetry plane is approached, and the peak of the mean Nusselt number occurs at $y=0.5$. Due to the presence of intensive convective flow in the $y$ direction, the convective heat transfer is enhanced, resulting in the appearance of two minor peaks at $y=0.2$ and 0.8 . 


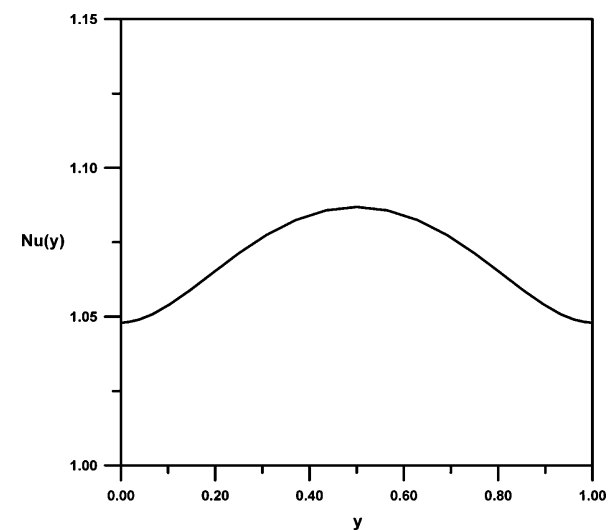

(a)

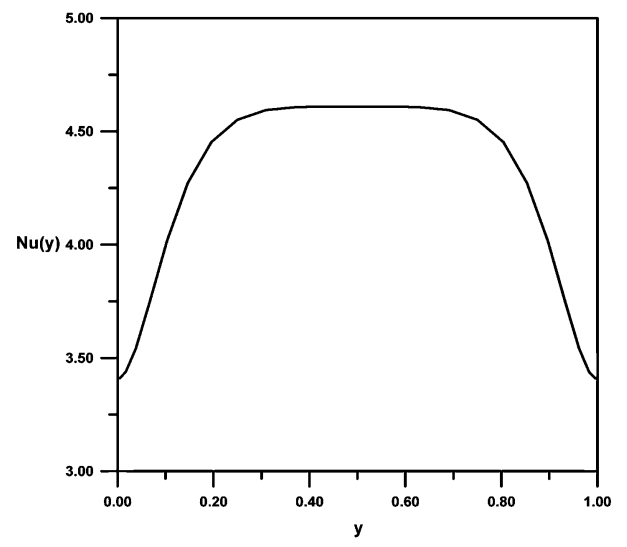

(c)

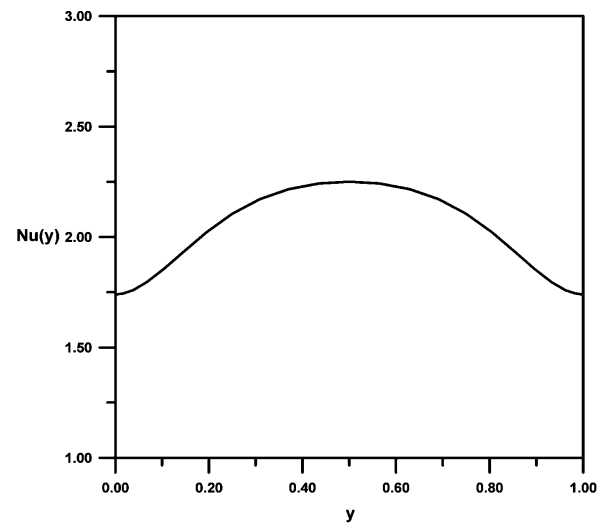

(b)

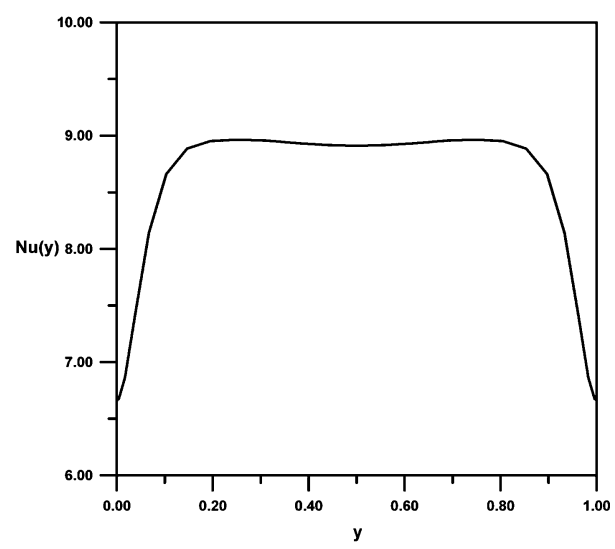

(d)

Figure 10. Distribution of the mean Nusselt number along the $y$ direction for $(a) \operatorname{Ra}=10^{3},(b) \operatorname{Ra}=10^{4}$, (c) $\mathrm{Ra}=10^{5},($ d $) \mathrm{Ra}=10^{6}$.

\section{CONCLUSIONS}

In the present numerical study, natural convection in a differentially heated cubic cavity has been studied using the Navier-Stokes equations in velocity-vorticity form and the energy equation. The governing equations have been solved by a coupled numerical algorithm based on the GDQ method. A grid independence study conducted for $\mathrm{Ra}=10^{3}$ and $10^{4}$ indicated that the accuracy of the numerical predictions of heat transfer parameters increases consistently with the mesh refinement. Validation test results obtained for $\mathrm{Ra}=10^{3}, 10^{4}, 10^{5}$, and $10^{6}$ show that the benchmark results could be achieved using only a $25^{3}$ mesh, which is much coarser compared to the finer grids used in other benchmark algorithms. As the Rayleigh number increases, the convective flow field is intensified near the end walls in the $y$ direction, which was confirmed by the appearance of two minor peaks of the mean Nusselt number. The thickness of the boundary layer near the isothermal walls 
decreases with increase in Rayleigh number. The results obtained demonstrate that the combination of the GDQ method and the velocity-vorticity form of the NavierStokes equations is an efficient numerical procedure to study flow and heat transfer in a differentially heated cubic enclosure.

\section{REFERENCES}

1. H. Fasel, Investigation of the Stability of Boundary Layers by a Finite-Difference Model of the Navier-Stokes Equations, J. Fluid Mech., vol. 78, pp. 355-383, 1976.

2. M. Napolitano and L. A. Catalano, A Multigrid Solver for the Vorticity-Velocity NavierStokes Equations, Int. J. Numer. Meth. Fluids, vol. 13, pp. 49-59, 1993.

3. G. Guj and F. Stella, A Vorticity-Velocity Method for the Numerical Solution of 3D Incompressible Flows, J. Comput. Phys., vol. 106, pp. 286-298, 1993.

4. C. Davis and P. W. Carpenter, A Novel Velocity-Vorticity Formulation of the NavierStokes Equations with Application to Boundary Layer Disturbance Evolution, J. Comput. Phys., vol. 172, pp. 119-165, 2001.

5. K. L. Wong and A. J. Baker, A 3D Incompressible Navier-Stokes Velocity-Vorticity Weak Form Finite Element Algorithm, Int. J. Numer. Meth. Fluids, vol. 38, pp. 99-123, 2002.

6. G. D. Mallinson and G. de Vahl Davis, Three-Dimensional Natural Convection in a Box: A Numerical Study, J. Fluid Mech., vol. 83, pp. 1-31, 1977.

7. O. Daube, Resolution of the 2D Navier-Stokes Equations in Velocity-Vorticity Form by Means of an Influence Matrix Technique, J. Comput. Phys., vol. 103, pp. 402-414, 1992.

8. M. S. Ingber and S. N. Kempka, A Galerkin Implementation of the Generalized Helmholtz Decomposition for Vorticity Formulations, J. Comput. Phys., vol. 169, pp. 215-237, 2001.

9. D. C. Lo and D. L. Young, Arbitrary Lagrangian-Eulerian Finite Element Analysis of Free Surface Flow Using a Velocity-Vorticity Formulation, J. Comput. Phys, vol. 195, pp. 175-201, 2004.

10. G. Guevremont, W. G. Habashi, and M. M. Hafez, Finite Element Solution of the Navier-Stokes Equations by a Velocity-Vorticity Method, Int. J. Numer. Meth. Fluids, vol. 10, pp. 461-475, 1990.

11. R. E. Bellman, B. G. Kashef, and J. Casti, Differential Quadrature: A Technique for the Rapid Solution of Nonlinear Partial Differential Equations, J. Comput. Phys., vol. 10, pp. 40-52, 1972.

12. C. Shu and B. E. Richards, Application of Generalized Differential Quadrature to Solve 2-Dimensional Incompressible Navier-Stokes Equations, Int. J. Numer. Meth. Fluids, vol. 15, pp. 791-798, 1992.

13. C. Shu, K. S. Yeo, and Q. Yao, An Efficient Approach to Simulate Natural Convection in Arbitrarily Eccentric Annuli by Vorticity-Stream Function Formulation, Numer. Heat Transfer A, vol. 38, pp. 739-756, 2000.

14. C. Shu, L. Wang, and Y. T. Chew, Numerical Computation of Three-Dimensional Incompressible Navier-Stokes Equations in Primitive Variable Form by DQ Method, Int. J. Numer. Meth. Fluids, vol. 43, pp. 345-368, 2003.

15. E. Tric, G. Labrosse, and M. Betrouni, A First Incursion into the 3D Structure of Natural Convection of Air in a Differentially Heated Cubic Cavity, from Accurate Numerical Solutions, Int. J. Heat Mass Transfer, vol. 43, pp. 4043-4056, 2000.

16. Y. Peng, C. Shu, and Y. T. Chew, A 3D Incompressible Thermal Lattice Boltzmann Model and its Application to Simulate Natural Convection in a Cubic Cavity, J. Comput. Phys., vol. 193, pp. 260-274, 2003. 
17. T. Fusegi, J. M. Hyun, K. Kuwahara, and B. Farouk, A Numerical Study of ThreeDimensional Natural Convection in a Differentially Heated Cubical Enclosure, Int. J. Heat Mass Transfer, vol. 34, pp. 1543-1557, 1991.

18. S. Wakashima and T. S. Saitoh, Benchmark Solutions for Natural Convection in a Cubic Cavity Using the High-Order Time-Space Method, Int. J. Heat Mass Transfer, vol. 47, pp. 853-864, 2004.

19. D. C. Lo, D. L. Young, and K. Murugesan, GDQ Method for Natural Convection in a Square Cavity Using a Velocity-Vorticity Formulation, Numer. Heat Transfer B, vol. 47, pp. 321-341, 2005.

20. C. Shu, Differential Quadrature and Its Application in Engineering, Springer-Verlag, London, UK, 2000. 
Copyright of Numerical Heat Transfer: Part B -- Fundamentals is the property of Taylor \& Francis Ltd. The copyright in an individual article may be maintained by the author in certain cases. Content may not be copied or emailed to multiple sites or posted to a listserv without the copyright holder's express written permission. However, users may print, download, or email articles for individual use. 